\title{
Palma y la Generación del 50. -I- Comenzando por una apostilla: el vano juicio de Sebastián Salazar Bondy sobre la Lima de Ricardo Palma
}

\author{
Harry Belevan-McBride \\ Academia Peruana de la Lengua \\ hbelevan@unmsm.edu.pe
}

\section{Resumen}

El presente ensayo es un trabajo en curso que aborda la relación conflictual de los autores emblemáticos de la denominada Generación del 50 con la ciudad capital. Esto tiene relevancia por cuanto, según los más destacados estudiosos de aquel período crucial de la literatura peruana del siglo XX, es con esa prole de escritores que se inicia, o cuando menos toma forma orgánica, la llamada literatura urbana. Sus múltiples aproximaciones serán entonces cotejadas con la visión de Lima que encontramos en las Tradiciones Peruanas de Ricardo Palma.

Palabras Clave: Generación del 50, Literatura peruana, siglo XX, Lima, Ricardo Palma.

\section{Abstract}

This work-in-progress delves into the conflictual relationship between noteworthy authors of the so-called Generation-50 and Peru's capital. This is relevant inasmuch as, according to most reliable sources on this crucial phase of $20^{\text {th }}$-Century Peruvian literature, those are the writers who shaped what is commonly referred to as urban literature. Their manifold approach shall contextualize Ricardo Palma's vision of Lima to be found in his Tradiciones peruanas.

Keywords: Generation-50, Peruvian literature, 20 ${ }^{\text {th }}$-Century, Lima, Ricardo Palma. 
Harry Belevan-McBride, escritor, diplomático y docente. Autor de una docena de libros en los géneros del cuento, la novela, el ensayo y el teatro. Es Doctor Honoris Causa de la Universidad del Havre, Francia, Académico de Número de la Academia Peruana de la Lengua y miembro del Instituto Ricardo Palma y de la Sociedad Peruana de Derecho Internacional. Ha sido profesor contratado en diversas universidades peruanas y extranjeras. Como diplomático, entre otros cargos se ha desempeñado como embajador del Perú en Francia, Portugal, Bolivia, Eslovenia, Austria, Mónaco y Eslovaquia, y ante la Unesco y las Naciones Unidas en Viena. Es actualmente Director del Instituto Raúl Porras Barrenechea de la Universidad Nacional Mayor de San Marcos. 
La literatura de la ciudad o literatura urbana es la que por primera vez aborda frontalmente, con crudo realismo y agudeza crítica, las deambulaciones del peruano vulnerable en la gran urbe -que sólo hay una, Lima-y, en algunos casos, en las ciudades más pobladas de provincias que también se convierten, así, en el tránsito (contra)natural de lo rural a lo urbano, es decir, del campo a la ciudad. La Generación del 50, o G-50 como hemos de referirnos indistintamente a dicha generación, retrata y explota esa mutación humana que ha de implicar desgarramientos emocionales en el nuevo habitante citadino. Tales transformaciones van a ir creando a su vez determinados enfrentamientos sociales, tanto somáticos como sicológicos. Estos rencores comenzarán a forjar una nueva tipología del ser peruano $^{1}$ derivada de la segregación del "limeño de pura cepa", como se pavonea el capitalino acriollado, hacia el "provinciano rústico", por llamarlo de alguna forma didáctica, y de la consiguiente aversión de este hacia aquel y "su" ciudad, un aldeano antropológicamente distinto al que habrá sido reflejado hasta entonces en nuestra literatura. Se trata de un individuo que vive una otredad desfasada respecto de terceros pero sobre todo de sí mismo, pues es un sujeto metamorfoseado desde su pasado y trasmutado a una condición de injerto -porque no llega a ser ni inserto ni integrado sino, a lo sumo, incrustadoen la gran ciudad, con toda su carga traumática de desencanto dentro del nuevo tejido social en el que ha de desenvolverse ${ }^{2}$.

l Decimos "del ser peruano" y no sólo del aldeano -provinciano o paisano--, porque hay en todo el proceso socio-histórico contemporáneo una influencia recíproca de las idiosincrasias limeña y provinciana.

2 La segregación no es sólo un fenómeno del tránsito campo-ciudad. En un informe de noviembre del 2017, la Unicef ha advertido de la desigualdad en la atención sanitaria y educativa de la niñez y juventud indígenas y mestizas (curiosos vocablos pero de uso oficial para designar a las poblaciones amazónicas y serranas, respectivamente). Más allá de cualquier extrapolación antojadiza el estudio, no obstante, estaría sugiriendo discriminaciones instintivas del inconsciente colectivo, propias de substratos étnicos ajenos a la división ancestral entre Lima y provincias, entendidas estas equívocamente como un conjunto uniforme. 
Luego de una somera exposición de la visión múltiple sobre la capital por parte de los principales autores de la G-50, se ha de cotejar esa visión caleidoscópica con la mirada igualmente fecunda de la Lima poscolonial que aportan las crónicas tradicionistas de Palma. Finalmente el estudio recalará, no tanto en una recapitulación abrazadora de la capital sino, más bien, en la dinámica evo-invo/lutiva que pudiera haber desembocado en una literatura con posible voluntad sintetizadora de una nueva metrópoli pero que resultará siendo, más bien, una síntesis inexorablemente desengañada, hostil, violenta y sobre todo humanamente descastada, que es imposible reconocer cuando se la evalúa con el sumario palmista. De donde, en primer lugar, la revisión de aquella acusación apostillada de Sebastián Salazar Bondy, uno de los autores más simbólicos de la G-50, cuyo vano juicio inculpa a Ricardo Palma de prolongar en la Lima republicana una arcadia colonial.

Los siguientes capítulos abordarán, así, otras tres temáticas igualmente puntuales:

II - Continuando con una anécdota: Luís Loayza y su nostalgia macilenta de la Lima de Ricardo Palma III - Prolongando la apostilla y la anécdota: el desencanto añorante de Julio Ramón Ribeyro con la Lima de Ricardo Palma IV - Sin paliativos ni atenuantes: Mario Vargas Llosa, o la implacable radiografía de la Lima de Ricardo Palma

Como no podría ser de otra forma, el presente primer capítulo del proyecto se nutre fundamentalmente de los planteamientos que formulé en mi discurso de ingreso al Instituto Ricardo Palma $^{3}$, y que consistió en un primer atisbo al juicio severo

3 Ver: Belevan-McBride, Harry (2013) Primeros apuntes sobre la Lima de Palma en las lecturas de Porras y Salazar Bondy, en: Aula Palma XII, pp.27-43. Instituto Ricardo Palma, Lima: Editorial Universitaria de la Universidad Ricardo Palma. 
que sobre Palma expresó en su momento Salazar Bondy. Este fue posiblemente el ensayista de mayor envergadura de la G-50 y, sin duda, la figura que emblematizó específicamente el ensayo acerca de Lima en la misma forma como Vargas Llosa lo personifica en la ficción: en ambos, con una visión despiadada y a menudo violenta de la capital. La usurpación de un escrito anterior nuestro es para sustraer aquel trabajo primigenio de su formalidad discursiva para que pueda integrarse, prolongándose, en los ensayos que dedicaremos ulteriormente a los sucesivos autores más representativos de esa generación, conforme con el esquema planteado líneas arriba.

\section{$-\mathrm{A}-$}

Lima es, qué duda cabe, una fuente inagotable de inspiración para todas las artes y en todos los tiempos. De Santa Rosa a Pardo y Aliaga y desde la Plaza Mayor de Rugendas que debió cruzar la Perricholi para dirigirse al puente y la alameda de Chabuca, a la Lima del Virrey Amat o de Segura, o bien de la Quinta Heeren de Humareda y La Placera acuarelada de Pancho Fierro; la capital del Perú ha sido desde hace prácticamente medio milenio la referencia constante, para el halago o el denuesto, de cuantos nacieron, moraron o transitaron en ella.

Pero, a fin de circunscribir el vasto panorama de lo que han sido los intentos por dilucidar aquel espíritu limeño durante estos primeros cuatro siglos de la capital, debemos ceñirnos a la época contemporánea y más ajustadamente al siglo XX, que es cuando se construye la limeñidad literaria definitiva de Lima. Así, el sarcasmo costumbrista de Leonidas Yerovi; la altivez cerebral de Riva Agüero; la presuntuosa veneración de Valdelomar; la disección afectiva de Raúl Porras y José Gálvez; el impasible desencanto de Ribeyro; la depurada violencia de Reinoso; el desaliento imperturbable de Luis Loayza; la mirada impulsiva de Vargas Llosa y el despecho quirúrgico 
de ese cirujano anatomista que fue Salazar Bondy; son todos elementos tributarios de la picardía humorística -aunque exenta de adulación-, de la sonrisa querendona -si bien nunca zalamera- y de la crítica aterciopelada -en son de mordacidad-, con que don Ricardo Palma miró, admiró y juzgó a su ciudad natal. Porque todos supieron, entonces como ahora, incluyendo a esa multitud de peruanos de hoy que banalmente posa una mirada de escarnio prejuiciado sobre Lima, que esta ciudad multisecular, como no ocurre con ninguna otra de nuestra vasta geografía, ha personificado lo que es el Perú y lo que ha sido por casi medio milenio, en todos sus claroscuros y complejidades pero, también, en toda su magnificente dignidad como nación.

Para razonar esta propuesta es necesario, sin embargo, acordar algunos criterios básicos, el primero de los cuales es convenir en lo que debe entenderse por generación, que es el punto de inflexión y de rencuentro del presente ensayo en cuanto procura contrastar dos generaciones distintas y distantes. Resulta difícil comprender cabalmente el significado ontológico del concepto mismo del vocablo generación: ¿cómo se mide el espacio-temporal continuo entre generaciones?; ¿cómo se evitan traslapos en/del calendario?; ¿cuál es el propósito histórico conclusivo de dar cuerda a ese péndulo que oscila en un vaivén tan similar al tictac acompasado de un reloj pero que, lejos de zanjar la indagación, más bien la encasilla fragmentándola, seccionando la noción misma de generación? En cambio, sí puede entenderse, acaso, el sentido de generación para establecer, por ejemplo, determinadas estadísticas demográficas o para facilitar la tarea de los registros públicos, y hasta podría encontrarse alguna utilidad para aquella curiosa disciplina científica llamada sociología. Pero, para regular los periodos literarios ordenándolos secuencialmente sin algunos principios elementales, el sistema de generación puede despistar más fácilmente que orientar. 
Por supuesto que, más allá de los aportes fundacionales de Karl Mannheim, los planteamientos de José Ortega y Gasset, considerados como el canon formal hispanoamericano sobre el régimen de las generaciones, son convincentes por su detallismo filosófico, al igual que las precisiones de Julián Marías (1949) en su libro intitulado, justamente, El método histórico de las generaciones. $\mathrm{Y}$ en lo que atañe a los estudiosos nacionales, las propuestas de expertos como Jorge Puccinelli, Ricardo González Vigil y Alberto Varillas amplían las aproximaciones iniciales de Jorge Luis Recavarren y César Pacheco Vélez, considerados los pioneros peruanos sobre la materia; (creemos, en cambio, que poco es el aporte de Carlos García Bedoya con su teoría sobre la periodización, por la algo arbitraria selección de los momentos de la literatura peruana $)^{4}$. Aun así, es difícil entender plenamente cómo es que el concepto de generación explicaría mejor que otros criterios la historia de las ideas o tendencias, y por qué esta heurística del método de la periodización nos revelaría las semejanzas o comparticiones entre las obras de un determinado grupo de creadores en una determinada circunstancia histórica, mejor que como lo harían los clásicos “-ismos" teóricos tales como el romanticismo, el vanguardismo, el indigenismo, el realismo, el naturalismo y la restante taxonomía definitoria con que solemos explicarnos la literatura y determinados conceptos culturales, más allá de las edades naturales de los escritores o de las fechas de aparición de sus obras.

Hechos estos alcances, repasemos ahora a la G-50 y la visión sobre Lima de sus más conspicuos representantes, a fin de poder contrastar dicha visión con la de nuestro egregio tradicionista. Lo primero que se observa es que hay una cierta discrepancia en la denominación misma: para algunos estudiosos esa generación

4 García-Bedoya Maguiña, Carlos: Para una periodización de la literatura peruana; Lima, ediciones de la Universidad Nacional Mayor de San Marcos, Serie Humanidades, 2004. 
debería llamarse, más bien, Generación del 45. Puccinelli, por ejemplo, refiriéndose a la G-50 señala que la integran los escritores nacidos después de la primera gran conflagración y que se dan a conocer con el retorno de la democracia a fines de 1939. Esta opinión es refrendada y acotada por Ricardo González Vigil cuando sostiene que convendría llamarla Generación del 45, por tratarse de un año significativo en lo nacional e internacional. Es pertinente recordar que este criterio se aplicó a la Generación del Novecientos o arielista -bisagra misma del siglo XX- y a la del Centenario o Generación del Conversatorio -cohesionada por el centésimo aniversario de la fundación de la república. Sin embargo, bien a pesar de estos y otros sólidos argumentos, a ese importante grupo de escritores y artistas de la mitad del siglo pasado se le conoce y reconoce como la Generación del 50 más que por cualquier otro nombre.

Pero hay otra divergencia adicional referida a los resultados de esta periodización, es decir, a las obras mismas de los escritores de la G-50. Para la mayoría de los estudiosos esta generación es la fundadora de lo que se conoció como Nueva Narrativa, cuyo acierto principal será el llamado neo-realismo urbano que llega a definir el cuento y la novela más allá del lenguaje 'regionalista' de los indigenistas, según Estuardo Núñez, a fin de retratar la lucha y la miseria, pero también las esperanzas e ilusiones de esa amalgama humana de la que ha de nacer el homo limensis. Esta aleación urbana representa un nuevo sujeto social tal como se ha dicho, producto primero de la marginación resultante de su inserción y, paulatinamente, de su incrustación en el entorno. Esta suerte de "inclusión excluyente" es el paso inmediato previo a la integración y la formación de la pequeña burguesía y de la clase media limeñas -el mestizaje en todas las vertientes imaginables de la palabra-, en cuanto que aquellas comparten determinadas afinidades sociales y económicas así como ciertos preceptos culturales, que habrán de funcionar como derivados naturales de la ecuación provincia-ciudad. 
Podría decirse, entonces, que la aparición del neo-realismo urbano con la narrativa de los integrantes de la G-50, marca el inicio del protagonismo literario de esos estratos socialmente marginales que, transformados, sobreviven día a día en las ciudades y fundamentalmente en la urbe limeña. Son aquellos arquetipos humanos que personifican desde el vendedor ambulante al oficinista asalariado; del pequeño propietario garajista al cachuelero o a la empleada doméstica; del sujeto polifuncional o "mil-oficios", para decirlo con este otro peruanismo tan ilustrativo, al cagatintas tramitador o, simplemente, al desempleado intentando abrirse trocha en la gran urbe a como dé lugar, a menudo delinquiendo. La aludida divergencia -con vientos de polémica, por cierto, por más que se pretendiera simplificarla - estriba en el cuestionamiento a esta aproximación por parte de quien, como Miguel Gutiérrez, fue tal vez el que más pretendió analizar desde la vereda exclusivamente política el aporte artístico de la G-50. Efectivamente, en su libro intitulado frontalmente La Generación del 50: un mundo dividido ${ }^{5}$, el reconocido autor de El viejo saurio se retira, si bien sostiene por un lado que el concepto de generación permite analizar nuestras peculiaridades como nación, también advierte que, si dicho concepto violenta la naturaleza de lo que se conoce en política como clase social y lucha de clases, el uso del concepto de generación resulta restringido y falseador. Restringido por subjetivo o falseador entendido como deformante, el hecho es que la gran mayoría

5 Este libro es, muy lamentablemente, un ensayo apologético del discurso vertical, como llamamos a los diktats hegemónicos, inherente al integrismo estereotipado del terrorismo en el Perú, apenas disfrazado con una advocación generacional. Hay en cambio tres otros estudios equilibrados sobre el tema: $L a$ Generación del 50 en la literatura peruana del siglo XX, trabajo colectivo publicado en 1996 por la Universidad de La Cantuta; "Apuntes sobre la Generación del 50 y Palma", de Roberto Reyes Tarazona aparecido en el 2017 en Aula Palma XV, y la tesis doctoral presentada el 2002 por Boniface Ofogo Nkama en la Universidad Complutense de Madrid, intitulada: La generación del 50 en el Perú (Una narrativa plural); desconocemos si este último trabajo ha sido publicado comercialmente. 
de estudiosos de la G-50 sí considera al neo-realismo urbano como el vehículo más idóneo para narrar el advenimiento de la clase media, a través de la representación inclemente de una coreografía humana ya no tanto rural sino más bien citadina, o en tránsito a serla. Los narradores más representativos de esa generación nos describen de manera elocuente en sus relatos a esos personajes de clase media: Carlos Eduardo Zavaleta, Enrique Congrains, Julio Ramón Ribeyro, Eleodoro Vargas Vicuña (con quien se terminará de archivar el indigenismo, según Estuardo Núñez), Sebastián Salazar Bondy, Antonio Gálvez Ronceros, Manuel Scorza, Oswaldo Reinoso y Mario Vargas Llosa, entre los principales ${ }^{6}$. De todos los narradores nombrados, González Vigil estima que Zavaleta fue el fundador de esa corriente neo-realista aunque, según confesión del propio Zavaleta, él no se consideró nunca ni guía ni cabecilla generacional sino, cronológicamente, apenas el primero en intentar un resuelto distanciamiento con el indigenismo.

Sin embargo, aun cuando González Vigil (1991: 375) cree acertadamente que Zavaleta es un autor-pivote del neo-realismo, también estima que Sebastián Salazar Bondy es "el escritor más completo de la Generación del $50 \ldots$ y... el más fecundo y polémico...", aunque se cuidará de precisar que, "sus poemas, narraciones, piezas dramáticas, artículos y ensayos, siendo con frecuencia de buena factura, no llegan a ser excepcionales ni magistrales..." ${ }^{7}$. No obstante, hay un libro de Salazar que destaca indudablemente dentro de la narrativa ensayística de

6 Cabría precisar que muchos de los poetas de esa generación reflejaron igualmente, y con encomiable sensibilidad lírica, los avatares de esa clase media; pero, precisamente por el caudal creativo que representan, este ensayo debe ceñirse a la narrativa de la Generación del 50 así como se limita a la prosa de Palma.

7 Este juicio parece justo cuando se compara la obra desigual de Salazar Bondy con la maestría narrativa de los cuentos de Ribeyro o de las novelas de Vargas Llosa. 
la G-50, aunque apareciera en los años sesenta: nos referimos al opúsculo llamado Lima la horrible. Se trata de un título suficientemente abrupto como para haberse convertido con el tiempo en una suerte de monótona locución interjectiva, muy en boga hasta hoy, para referirse a la capital, una expresión que escuchamos a menudo en boca de gentes que ni siquiera han leído el libro (y menos aún saben de quién proviene originalmente la expresión) pero que repiten el estribillo por mera inercia, o bien para mejor remarcar frívolamente su preferencia por el Perú profundo -invocación no exenta de algún pintoresquismo andino y hasta racista - frente a la supuestamente execrable Lima. Pero nos detenemos en este libro porque no es sólo el texto más trascendente entre toda la obra de Salazar Bondy sino que, de algún modo, se convirtió en un libro representativo de cierta actitud generacional frente a la realidad que se estaba viviendo en esos años. Y ello porque, sin ser una metáfora ficcional sobre Lima, representó literariamente la reflexión más frontal de esa G-50 aunque también, acaso, la más envejecida si se la mira en el retrovisor del medio siglo transcurrido.

Si la premisa es válida se está reforzando, entonces, la propuesta según la cual hablar de lo urbano es referirnos fundamentalmente a Lima pues la capital encarna en simultáneo, a partir precisamente de los años cincuenta, el explosivo conglomerado de la esperanza y de la frustración ciudadanas mayoritarias. Estas, sin embargo -y he aquí lo excepcional—, se irán vaciando progresivamente de los malabares de esa lucha de clases postulada por Miguel Gutiérrez (1988). La esperanza en que "la migración hacia Lima servirá para encontrar trabajo y una vida mejor" el todo entrecomillado como que es una reflexión tantas veces escuchada-, se disuelve con la frustración y desengaño ante la obscenidad cotidiana a la cual, y a pesar de todo y contra todo, se acomoda el provinciano dentro de la gran ciudad, en lugar de aventurarse en un suicidio ideológico colectivo como el que le susurra un idealismo político alcanzable mediante el 
terror. Habrá, sí, un microcosmos migratorio en otros espacios urbanos del país como, por ejemplo, con el desplazamiento de poblaciones puneñas y circunlacustres hacia Arequipa, pero la lente neo-realista estará literariamente enfocada en Lima.

Muchos de los personajes en los relatos de Zavaleta, Ribeyro o Vargas Llosa son seres irremediablemente humillados, o cuando menos mediocrizados por el entorno cotidiano de esa Lima de entonces en la que, según Salazar, debía agonizar la supuesta utopía palmista. Sin embargo, hay quienes no lo consideran así y creen, más bien, que la Lima decimonónica sobrevive hasta ahora entorpeciendo el surgimiento de una nueva ciudad, a través de una clase social particular que portaría la antorcha supuestamente inextinguible de la lucha de clases. Lima la horrible de Sebastián Salazar Bondy sintetiza el espíritu de esa propuesta, a juicio nuestro sesgada, de ciertos autores de la G-50. A pesar de ello, la realidad de este medio siglo transcurrido desde la aparición del citado ensayo ha impugnado por completo aquellos planteamientos, puesto que la insurrección anhelada por algunos teóricos no logró nunca impregnar a lo que comúnmente se tilda de masas populares, cuyo metabolismo biológico se adaptó para estar en condiciones de alcanzar y consumir un determinado progreso material, drenando al mismo tiempo toda intención de sedición social o de cualquier otro impedimento que pudiese trabar el acceso de esas masas a los beneficios, reales e ilusorios a la vez, de la modernidad.

Si el ADN de la Generación del 50 es la transición del campo a la ciudad en nuestra literatura, mencionar lo urbano es entonces, como ya se ha repetido, hablar fundamentalmente de Lima; por eso, discutir sobre esta generación es, de algún modo, discurrir sobre la gran urbe peruana. Ahora bien; al hacerlo podría decirse que, literaria y socialmente, hay en Lima dos ciudades emblemáticas que se confrontan desde la 
Colonia hasta la primera mitad del siglo XX, es decir, durante esos más de cuatro siglos que van de la fundación española al surgimiento, precisamente, de la G-50. De ser así, parecería entonces que los dos extremos de la visión limeña en ese lapso secular son Ricardo Palma y Sebastián Salazar Bondy, como antípodas de esa limeñidad referida al inicio. Hay por cierto otras miradas igualmente significativas de la capital peruana, de escritores y viajeros tan transcendentes como Palma y más que Salazar: las del padre Bernabé Cobo o de Paul Morand hasta las miradas del Jaguar o Zavalita que convierten al Leoncio Prado y La Catedral en maquetas a escala de la propia capital, pasando por la Lima relatada por José Gálvez o Raúl Porras. Pero, con lugar a pocas dudas, las dos representaciones paradigmáticas de Lima serían las de Ricardo Palma y Sebastián Salazar Bondy: ambas republicanas, la visión de Palma es evocadora de unas tradiciones que rememoran a menudo la afable Lima colonial -aunque el tradicionista sería también "el intérprete del medio pelo", según la expresión admirativa de Mariátegui en sus 7 Ensayos...-, mientras que la capital peruana vista por Salazar es una Lima simplemente horrible; y esto, porque Salazar embiste frontalmente a la ciudad contemporánea acusándola de perpetuar, con y a través de Palma, una arcadia colonial. La mejor síntesis de la insensible subjetividad de este juicio sobre Lima, es la del reconocido crítico y estudioso Julio Ortega:

La condena de Salazar Bondy está hecha en un lenguaje del archivo discursivo de la noción de Lima como falso centro del país. Es un ensayo más literario que analítico y, por eso mismo, una metáfora deliberadamente anacrónica de su tema (2010: 15)

Es a partir de esta aserción que desarrollaremos más adelante el segmento -B- y último del presente capítulo, correspondiente a este primer ensayo sobre Palma y la Generación del 50. 


\section{Bibliografía}

Belevan-McBride, H. (2013) Primeros apuntes sobre la Lima de Palma en las lecturas de Porras y Salazar Bondy, en: Aula Palma XII, pp.27-43. Lima: Instituto Ricardo Palma, Editorial Universitaria de la Universidad Ricardo Palma.

García-Bedoya Maguiña, C. (2004) Para una periodización de la literatura peruana; Lima, ediciones de la Universidad Nacional Mayor de San Marcos, Serie Humanidades.

González Vigil, R. (1991) El cuento peruano. 1942-1958. Lima: Ediciones Copé

Gutiérrez, M. (1988) La Generación del 50: un mundo dividido. Historia y balance. Lima: Ediciones Sétimo Ensayo 1

La Generación del 50 en la literatura peruana del siglo XX, (1996) Lima: Universidad de La Cantuta.

Loayza, L. (2010) Relatos. Prólogo de Julio Ortega, p. 15. Lima: Editorial Universitaria de la Universidad Ricardo Palma.

Marías, J. (1949). El método histórico de las generaciones. Madrid: Revista de Occidente.

Ofogo Nkama, B. (2002) La generación del 50 en el Perú (Una narrativa plural). Tesis doctoral. Universidad Complutense de Madrid.

Reyes Tarazona, R. (2017). Apuntes sobre la Generación del 50 y Palma, en Aula Palma XV. Lima: Editorial Universitaria de la Universidad Ricardo Palma. 